\title{
Damping profile of standing kink oscillations observed by SDO/AIA
}

\author{
D. J. Pascoe ${ }^{1}$, C. R. Goddard ${ }^{1}$, G. Nisticò ${ }^{1}$, S. Anfinogentov ${ }^{2}$, and V. M. Nakariakov ${ }^{1}$ \\ ${ }^{1}$ Centre for Fusion, Space and Astrophysics, Department of Physics, University of Warwick, CV4 7AL, UK \\ e-mail: D. J.Pascoe@warwick . ac.uk \\ ${ }^{2}$ Institute of Solar-Terrestrial Physics, Lermontov st. 126a, 664033 Irkutsk, Russia
}

Received 25 November 2015 / Accepted 18 December 2015

ABSTRACT

\begin{abstract}
Aims. Strongly damped standing and propagating kink oscillations are observed in the solar corona. This can be understood in terms of mode coupling, which causes the wave energy to be converted from the bulk transverse oscillation to localised, unresolved azimuthal motions. The damping rate can provide information about the loop structure, and theory predicts two possible damping profiles.

Methods. We used the recently compiled catalogue of decaying standing kink oscillations of coronal loops to search for examples with high spatial and temporal resolution and sufficient signal quality to allow the damping profile to be examined. The location of the loop axis was tracked, detrended, and fitted with sinusoidal oscillations with Gaussian and exponential damping profiles.

Results. Using the highest quality data currently available, we find that for the majority of our cases a Gaussian profile describes the damping behaviour at least as well as an exponential profile, which is consistent with the recently developed theory for the damping profile due to mode coupling.
\end{abstract}

Key words. magnetohydrodynamics (MHD) - Sun: atmosphere - Sun: corona - Sun: magnetic fields - Sun: oscillations - waves

\section{Introduction}

Observations of kink oscillations in the solar corona date back to the Transition Region And Coronal Explorer (TRACE) in the late 1990s, when strongly-damped transverse displacements of coronal loops were detected after flare events (Aschwanden et al. 1999; Nakariakov et al. 1999). Improving on previous studies, recent high-resolution images from the Atmospheric Imaging Assembly (AIA) of the Solar Dynamics Observatory (SDO) have permitted us to detect new details concerning kink wave dynamics (e.g. Aschwanden \& Schrijver 2011; White \& Verwichte 2012). In contrast to the standard decaying oscillations, a new class of low-amplitude and decayless oscillations has been discovered, which is unrelated to any impulsive energy release (Nisticò et al. 2013; Anfinogentov et al. 2013). A recent study demonstrates that this new regime is commonly present in active regions (Anfinogentov et al. 2015). A better understanding of the excitation mechanisms for kink oscillations motivated Zimovets \& Nakariakov (2015) to perform a large-scale investigation using SDO/AIA. A statistical study over 58 catalogued events demonstrates that high-amplitude decaying kink oscillations of coronal loops are preferentially excited by low-coronal eruptions rather than blast-waves launched by flares. An analysis of the damping profile over 52 events from the same catalogue (Goddard et al. 2016) shows that almost half of the events exhibit an oscillation better approximated by a non-exponential envelope, instead of a pure exponential profile.

The strong damping of flare-excited standing kink modes observed by TRACE was described by Ruderman \& Roberts (2002) and Goossens et al. (2002) in terms of resonant absorption, i.e. the coupling of transverse kink motions to azimuthal Alfvén waves in loops that have a finite inhomogeneous layer between the high density (low Alfvén speed) core and low density (high Alfvén speed) background. Pascoe et al. (2010) applied this mechanism to propagating kink waves observed to be strongly damped when propagating along loop structures (Tomczyk \& McIntosh 2009).

Pascoe et al. (2012) performed numerical simulations of strongly damped kink waves and found that the damping behaviour for early times is best described by a Gaussian profile. Hood et al. (2013) produced an analytical description the damping behaviour for all times in the form of an integro-differential equation for the continuous variation of the amplitude, confirming that the initial (non-asymptotic) stage of the damping envelope is well approximated by a Gaussian profile, and the later (asymptotic) stage by an exponential profile. The switch between profiles depends on the loop density contrast ratio and occurs sooner (later) for larger (smaller) contrasts (Pascoe et al. $2013)$. The signal quality $(\tau / P)$ depends on the loop density contrast and width of the inhomogeneous layer (see also reviews by Pascoe 2014 and De Moortel et al. 2016). Analysis of broadband kink oscillations (Verth et al. 2010) confirms that the damping rate was frequency dependent but the data is too noisy to distinguish between Gaussian and exponential profiles (Pascoe et al. 2015).

In Sect. 2 we describe the observations of damped standing kink modes using SDO/AIA. In Sect. 3 we investigate the suitability of Gaussian and exponential profiles to describe the observed damping behaviour. Discussion and conclusions are presented in Sect. 4.

\section{Observations by SDO/AIA}

Kink oscillation events were selected from Table 1 in Goddard et al. (2016). A large number of events from the catalogue need to be excluded as unsuitable for our purposes in this paper. Investigating the damping profile requires that the loop be accurately identified and tracked for several consecutive cycles once 

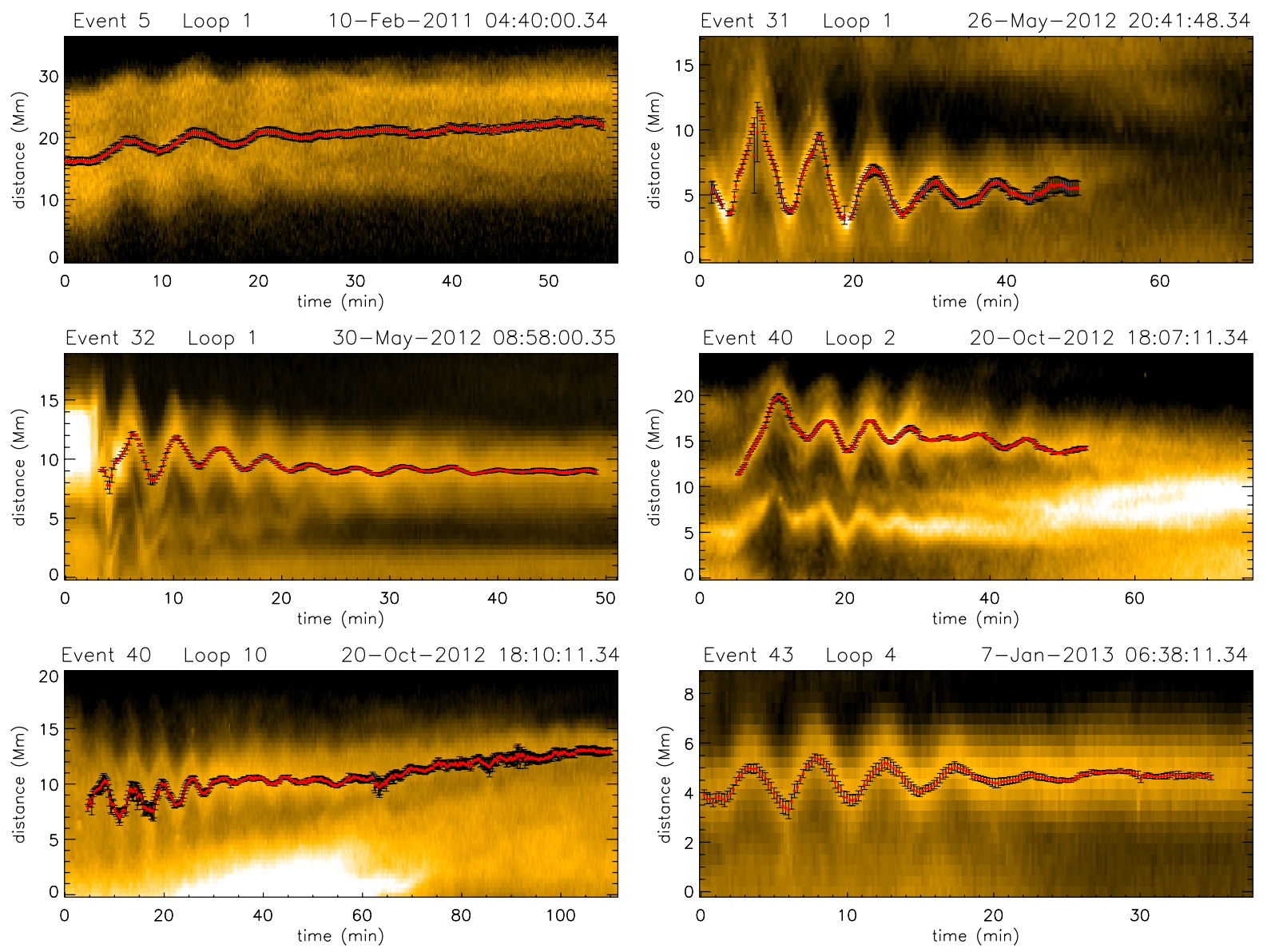

Fig. 1. Time-distance maps for selected events from Goddard et al. (2016). The symbols (with error bars) represent the locations of the loop axis.

the oscillation begins with a period of oscillation that remains stable.

Time-distance (TD) maps for suitable events were created with SDO/AIA EUV $171 \AA$ Aata (spatial resolution of 0.6 arcsec per pixel and temporal cadence of $12 \mathrm{~s}$ ) by taking linear slits with a 5 pixel width perpendicular to the oscillating loop and stacking the intensities along the slit, averaged over the width to increase the signal-to-noise ratio in time. A series of 100 slits were created for each loop, perpendicular to an elliptical or linear fit of the loop (depending on loop orientation). The displacement of the loop axis by the global standing kink mode is greatest at the loop apex and decreases to zero at the loop footpoints. Oscillations are therefore generally best measured near the loop apex, though the particular details of the event such as line of sight effects can make a measurement in the loop legs more suitable. The slit, which maximised the clarity of the TD map and the apparent amplitude of the oscillation, was then chosen for further analysis.

Figure 1 shows the TD maps for the selected events. From the TD maps, the loop centre (symbols with error bars) is located by fitting the intensity with a Gaussian profile. The fit is carried out within a window around the loop of interest to avoid contamination from other loops or bright features. The error for the loop centre position is determined separately for each data point, according to the 1-sigma estimate reported by the Gaussian fit routine. The time series for the location of the loop centre as a function of time is then used to obtain the kink mode signal. The damping behaviour of this kink oscillation is investigated by fitting exponential and Gaussian envelopes as described in the following section.

\section{Fitting mode coupling damping profiles}

We consider the damping profile that best describes the observed decay of kink oscillations, considering in particular the two limiting cases predicted by mode coupling. The Gaussian damping profile was initially derived in the context of propagating (in z) waves, but the same behaviour applies to standing modes with the change of variable $t=z / C_{\mathrm{k}}$ (e.g. Sect. 6.1 of Pascoe et al. 2013). In principle, the analysis may be carried out by only considering the (absolute values of the) extrema of the oscillation, to which exponential and Gaussian profiles can be fitted. Equivalently, the natural logarithm of these extrema can be fitted with linear or quadratic functions (or determine a switch between such profiles). However, since our oscillations evidently contain background trends and there are a small number of cycles of oscillation observed, we maximise our data points by fitting the detrended oscillations with damped sinusoids of the form

$A \sin (\omega t-\phi) \exp \left(-t^{2} / 2 \tau_{\mathrm{g}}^{2}\right)$

for the case of the Gaussian damping profile, and

$A \sin (\omega t-\phi) \exp \left(-t / \tau_{\mathrm{d}}\right)$

for an exponential damping profile. The parameters $A, \omega, \phi$, and $\tau$ are determined by a Levenberg-Marquardt least-squares 
D. J. Pascoe et al.: Damped kink mode profiles
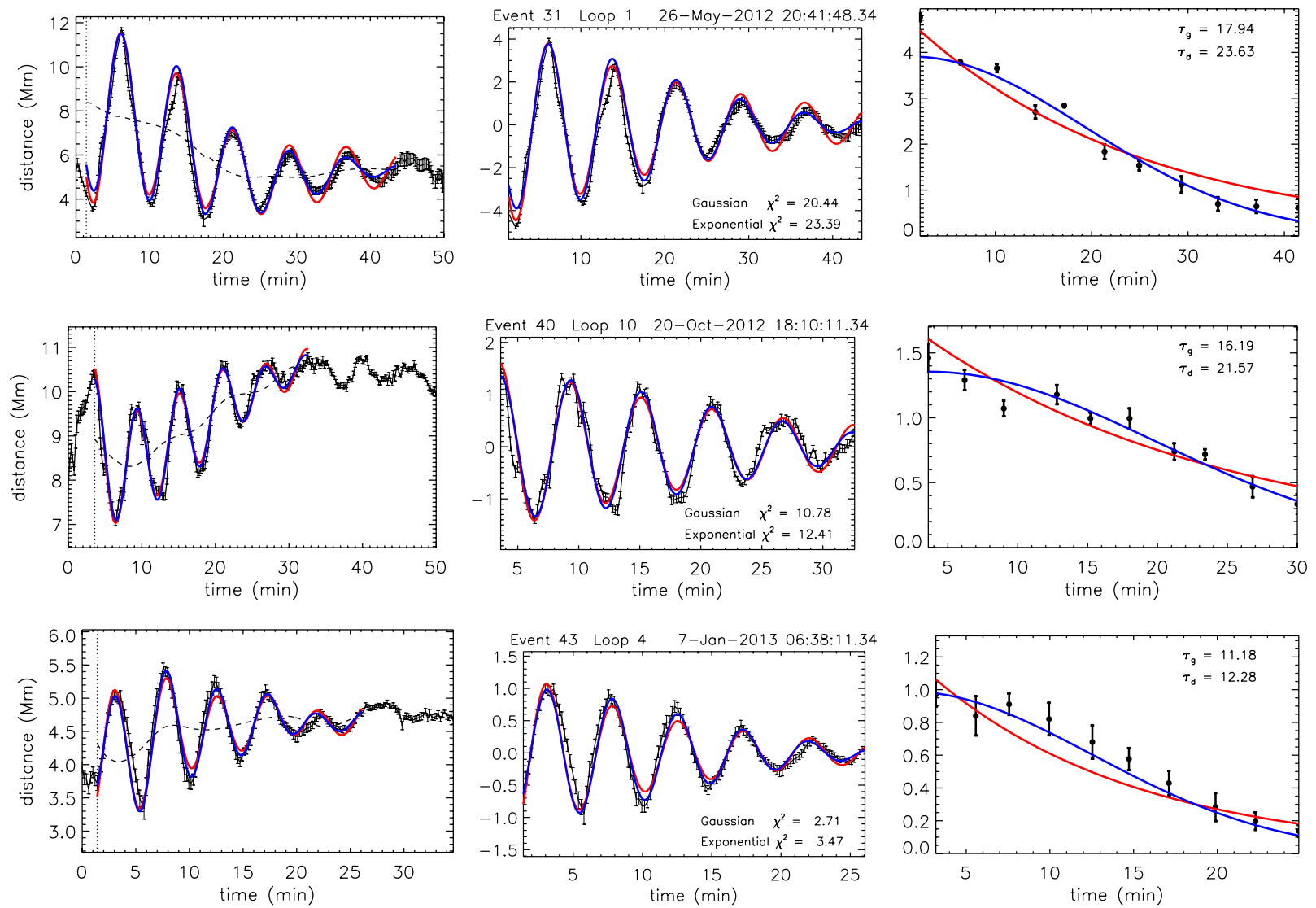

Fig. 2. Examples of damped kink oscillations for which a Gaussian envelope is found to be a better fit than an exponential envelope. The left panels shows the position of the loop axis as a function of time, fitted with sinusoids with a Gaussian (blue lines) and exponential (red lines) damping envelope. The vertical dotted lines and dashed lines denote the start time $t_{0}$ and the background trend, respectively. The middle panels show the detrended oscillations, while the panels on the right show the absolute values of the extrema with fitted envelopes.
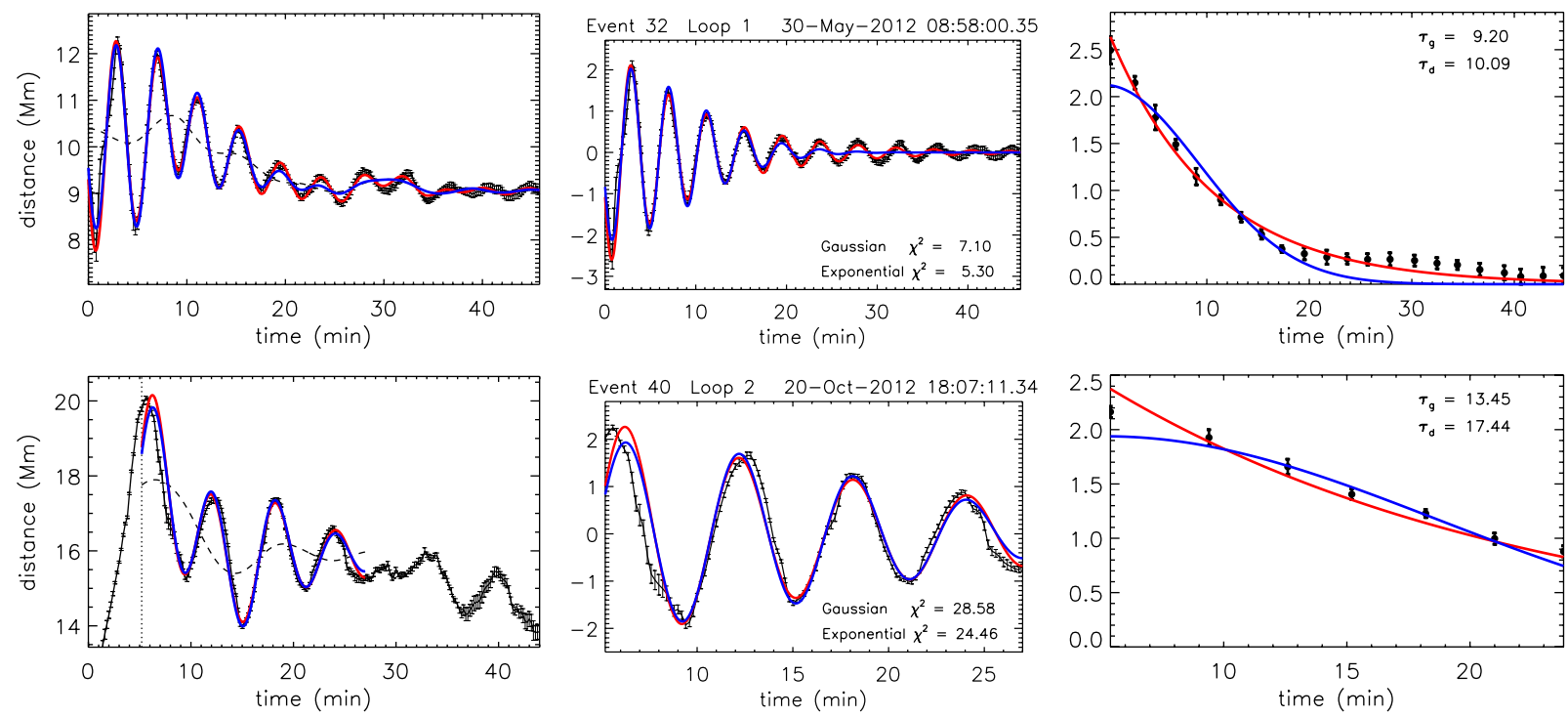

Fig. 3. Kink oscillations for which an exponential damping profile was found to be a better fit than a Gaussian profile. Line styles as in Fig 2.

fit with data points weighted according to their errors. The background trend is often taken to be a polynomial. For example White \& Verwichte (2012) use a third order polynomial for the background, while Aschwanden et al. (2002) contains examples of polynomial trends with order 1-6. Here the background trend is found by spline interpolation of the oscillation extrema, which ensures the best fit for the sinusoidal components of
Eqs. (1) and (2). The damped sinusoid fits for the selected events are shown in Figs. 2-4. The accuracy of the two damping profiles are compared by calculating their $\chi^{2}$ values and inspection of how well they describe the evolution of the oscillation. The fitted values of $\tau_{\mathrm{g}}$ and $\tau_{\mathrm{d}}$ are given in the right panels. The different models not only produce different damping times, but those times have different relationships to the physical parameters, e.g. 

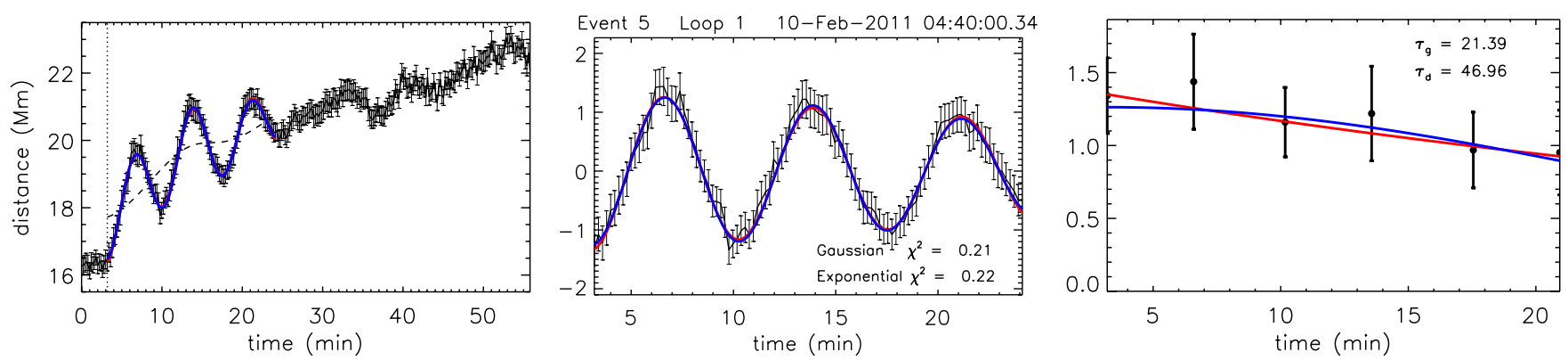

Fig. 4. Kink oscillation for which exponential and Gaussian damping profile fits are equivalent. Line styles as in Fig. 2.

$\tau_{\mathrm{g}} / \tau_{\mathrm{d}}=\epsilon^{\frac{1}{2}} \pi / 2$, where $\epsilon$ is the normalised inhomogeneous layer width.

We stress that the fitting is not applied to the entire time series, i.e. the oscillations have a defined start and end time. The end time is typically determined by the sinusoidal component of the fit becoming unsuitable, e.g. a significant change in period or phase implying the oscillation cannot be considered a single, continuous event. For example, in the bottom panel of Fig. 3 the loop experiences a large displacement from its initial position before it then begins oscillating. After approximately three cycles of oscillation there is a significant change in period and amplitude, which are not well described by either fitted profile. Later the displacement amplitude increases again and the period is similar to that at the beginning of the oscillation, although a significant phase shift suggests it is not a continuation of the original oscillation. Consequently, the fit is restricted to a short part of the time series.

Figure 2 shows examples of oscillations for which a Gaussian envelope is found to be more suitable than an exponential envelope. The fitted Gaussian profiles have smaller values of $\chi^{2}$ than the fitted exponential profiles and can be seen to describe the evolution of the oscillation more accurately. This is the case in particular with regard to the damping rate during the first few cycles of the oscillation, which is where the Gaussian and exponential profiles differ the greatest. For comparison with the previous examples, Fig. 3 shows oscillations for which an exponential damping envelope was found to give a better fit than a Gaussian envelope, while Fig. 4 shows an example where the envelopes are found to be approximately equivalent.

\section{Discussion and conclusions}

The events considered in this letter were only selected on the basis of the quality of the data being suitable for determining the damping profile. Both Gaussian and exponential envelopes are consistent with damping due to mode coupling and accurate identification of the correct profile can provide additional information about the oscillating loop. Nonetheless, we demonstrate that in the majority of our cases (four out of six; Figs. 2 and 4) the Gaussian profile is at least as suitable in describing the data as an exponential profile.

Our requirement of the kink oscillation having a high signal quality is equivalent to weak damping. Mode coupling is weak if the density contrast ratio and/or inhomogeneous layer width are small. A small density contrast $(\$ 5)$, therefore, favours both a Gaussian damping profile (Pascoe et al. 2013) and high signal quality. An exponential damping profile is expected for loops with large density contrasts $(\gtrsim 5)$ but thin transition layers (to maintain a signal quality large enough to observe), which may be the case for the oscillations shown in Fig. 3.
The use of exponential and Gaussian damping profiles in this study is motivated by recent theoretical developments for the damping of kink oscillations by mode coupling. However, this is not the only mechanism capable of producing a non-exponential damping profile; for example Nakariakov et al. (2009) demonstrate a non-exponential profile of the kink oscillation amplitude evolution. However, in the context of using mode coupling to explain observed damping, and particularly for any subsequent seismological inversions based on this mechanism, our results demonstrate that it is not necessarily appropriate to assume the damping profile to be exponential, as proposed by recent numerical (Pascoe et al. 2012, 2013) and analytical (Hood et al. 2013) modelling. In particular, it should be taken into account in the scaling of the damping time with the oscillation period (Ofman \& Aschwanden 2002).

Acknowledgements. This work is supported by the Marie Curie PIRSES-GA2011-295272 RadioSun project, the European Research Council under the SeismoSun Research Project No. 321141 (DJP, CRG, VMN), the STFC consolidated grant ST/L000733/1 (GN, VMN), the Russian Foundation of Basic Research under grants 15-02-01089-a, 15-02-03835-a, and 15-32-20504 mol_a_ved, and the Federal Agency for Scientific Organisations base project II.16.3.2 "Non-stationary and wave processes in the solar atmosphere" (SA). The data is used courtesy of the SDO/AIA team.

\section{References}

Anfinogentov, S., Nisticò, G., \& Nakariakov, V. M. 2013, A\&A, 560, A107 Anfinogentov, S. A., Nakariakov, V. M., \& Nisticò, G. 2015, A\&A, 583, A136 Aschwanden, M. J., \& Schrijver, C. J. 2011, ApJ, 736, 102

Aschwanden, M. J., Fletcher, L., Schrijver, C. J., \& Alexander, D. 1999, ApJ, 520,880

Aschwanden, M. J., de Pontieu, B., Schrijver, C. J., \& Title, A. M. 2002, Sol. Phys., 206, 99

De Moortel, I., Pascoe, D. J., Wright, A. N., \& Hood, A. W. 2016, Plasma Physics and Controlled Fusion, 58, 014001

Goddard, C. R., Nisticò, G., Nakariakov, V. M., \& Zimovets, I. V. 2016, A\&A, in press, DOI: 10.1051/0004-6361/201527341

Goossens, M., Andries, J., \& Aschwanden, M. J. 2002, A\&A, 394, L39

Hood, A. W., Ruderman, M., Pascoe, D. J., et al. 2013, A\&A, 551, A39

Nakariakov, V. M., Ofman, L., Deluca, E. E., Roberts, B., \& Davila, J. M. 1999, Science, 285, 862

Nakariakov, V. M., Aschwanden, M. J., \& van Doorsselaere, T. 2009, A\&A, 502, 661

Nisticò, G., Nakariakov, V. M., \& Verwichte, E. 2013, A\&A, 552, A57

Ofman, L., \& Aschwanden, M. J. 2002, ApJ, 576, L153

Pascoe, D. J. 2014, Res. Astron. Astrophys., 14, 805

Pascoe, D. J., Wright, A. N., \& De Moortel, I. 2010, ApJ, 711, 990

Pascoe, D. J., Hood, A. W., de Moortel, I., \& Wright, A. N. 2012, A\&A, 539, A37

Pascoe, D. J., Hood, A. W., De Moortel, I., \& Wright, A. N. 2013, A\&A, 551, A40

Pascoe, D. J., Wright, A. N., De Moortel, I., \& Hood, A. W. 2015, A\&A, 578, A99

Ruderman, M. S., \& Roberts, B. 2002, ApJ, 577, 475

Tomczyk, S., \& McIntosh, S. W. 2009, ApJ, 697, 1384

Verth, G., Terradas, J., \& Goossens, M. 2010, ApJ, 718, L102

White, R. S., \& Verwichte, E. 2012, A\&A, 537, A49

Zimovets, I. V., \& Nakariakov, V. M. 2015, A\&A, 577, A4 